\title{
SIMULAÇÃO FÍSICA E MATEMÁTICA DA PENETRAÇÃO DE DE GÁS NA PERNA DE SUBIDA DE UM MODELO FÍSICO DE DESGASEIFICADOR RH*
}

Bernardo Martins Braga ${ }^{1}$

Leonardo Neves ${ }^{2}$

Roberto Parreiras Tavares ${ }^{3}$

\section{Resumo}

$\mathrm{O}$ desgaseificador $\mathrm{RH}$ é um reator de refino secundário bem estabelecido. Nesse equipamento, gás inerte é usado para promover a circulação de aço entre a panela de aciaria e uma câmara de vácuo. A predição correta da dispersão de gás na perna de subida do RH é importante para a modelagem confiável do processo. O presente trabalho estudou a penetração de gás na perna de subida do $\mathrm{RH}$ por meio de modelos físico e matemático. No estudo, foram considerados apenas um ou dois bicos injetores e vazões de gás, por bico, entre $5 \mathrm{l} / \mathrm{min}$ e $50 \mathrm{l} / \mathrm{min}$. Para as condições com um bico injetor, os resultados das duas abordagens mostraram boa concordância qualitativa. Já para as condições com dois bicos injetores, a concordância só foi satisfatória para vazões de até $301 / \mathrm{min}$ por bico. Adicionalmente, os resultados obtidos indicaram que o problema de penetração de gás em modelos matemáticos de desgaseificador RH identificado na literatura está relacionado com erros numéricos e não com falhas na modelagem da física do problema.

Palavras-chave: Desgaseificador RH; Modelo físico, Modelo matemático; penetração de gás.

\section{PHYSICAL AND MATHEMATICAL SIMULATION OF THE GAS PENETRATION IN THE UPPER LEG OF A PHYSICAL MODEL OF RH DEGASSER}

\section{Abstract}

The RH degasser is a well-established secondary refining reactor. In this equipment, inert gas is used to promote the circulation of steel between a steelmaking ladle and a vacuum chamber. The correct prediction of gas dispersion in the upper leg of $\mathrm{RH}$ is important to ensure a reliable modeling of the process. This work studied the gas penetration in the upper leg of $\mathrm{RH}$ by means of physical and mathematical models. Only one or two injectors were considered and gas flow rates, by injector, between $5 \mathrm{l} / \mathrm{min}$ and $50 \mathrm{l} / \mathrm{min}$ were employed. For cases with one injector, results from both approaches showed good qualitative agreement. On other hand, for cases with two injectors, the agreement was just satisfactory for gas flow rates up to $301 / \mathrm{min}$ by injector. Additionally, the obtained results showed that the issue of gas penetration in mathematical models of $\mathrm{RH}$ degasser identified in the literature is related to numerical errors and does not stem from inadequate modeling of problem physics.

Keywords: RH degasser; Physical model; Mathematical model; Gas penetration.

1 Engenheiro Metalurgista, Mestre, Doutorando, PPGEM, UFMG, Belo Horizonte, Minas Gerais e Brasil.

2 Engenheiro Metalurgista, Doutor, Professo Efetivo, Departamento de Materiais, CEFET-MG, Belo Horizonte, Minas Gerais, Brasil.

3 Engenheiro Metalurgista, PhD, Professor, Departamento de Metalurgia e Materiais, UFMG, Belo Horizonte, Minas Gerais, Brasil. 


\section{INTRODUÇÃO}

O desgaseificador $\mathrm{RH}$ é um reator de refino secundário bem-estabelecido na produção de aços com teores muito baixos de elementos intersticiais (carbono, nitrogênio, hidrogênio e oxigênio) [1-2]. Nesse processo, dutos refratários (pernas do $\mathrm{RH}$ ) conectados a uma câmara de vácuo são parcialmente submersos no aço líquido presente em uma panela de aciaria. $O$ aço líquido é circulado através das pernas do $\mathrm{RH}$ graças à injeção de gás por bicos posicionados em uma das pernas do reator.

Modelos físicos e matemáticos foram empregados na literatura para melhorar o entendimento e otimizar o processo $\mathrm{RH}$. No entanto, diversos modelos matemáticos falham na predição da penetração de gás na perna de subida do reator $\mathrm{RH}$, de modo que o gás permanece aderido às paredes da perna de subida do equipamento [3-6]. Esse fato é preocupante, pois põe em dúvida a validade desses modelos matemáticos para duas aplicações de interesse prático: previsão da taxa de circulação do aço pela câmara de vácuo - importante parâmetro de processo - e análise das solicitações mecânica e química dos refratários da perna subida. Também é comprometida a possibilidade de extensão desses modelos para previsão de fenômenos mais complexos que dependem da distribuição de gás no reator, como a descarburação e a flotação de inclusões não-metálicas [7].

A literatura apresenta apenas soluções ad-hoc para o problema de penetração de gás, como a prescrição do campo de fração volumétrica de gás ou da velocidade relativa entre fases [8]. O presente trabalho apresenta um modelo matemático capaz de prever a penetração de gás na perna de subida de gaseificadores $\mathrm{RH}$. Os resultados do modelo matemático foram confrontados com dados do modelo físico de um desgaseificador $\mathrm{RH}$ industrial.

\section{MATERIAIS E MÉTODOS}

Primeiramente, serão descritos os experimentos físicos realizados e em seguida será apresentado o modelo matemático utilizado.

\subsection{Experimentos em Modelo Físico}

Foi utilizado um modelo físico em escala 1:5 de um desgaseificador $\mathrm{RH}$ industrial. $\mathrm{O}$ modelo físico, construído em acrílico, utiliza aproximadamente 2001 de água para simular as 180 t de aço presentes na panela industrial. A Figura 1 apresenta as dimensões do modelo físico.

O modelo físico foi dimensionado utilizando critérios de similaridades baseados na igualdade dos números adimensionais de Froude e Froude modificado e na correção do volume de gás no $\mathrm{RH}$ industrial [4,9]. A vazão de ar no modelo físico $\boldsymbol{Q}_{\boldsymbol{M}}(\mathrm{l} / \mathrm{min})$ se relaciona com vazão de argônio no equipamento industrial $\boldsymbol{Q}_{I}(\mathrm{NI} / \mathrm{min})$ por:

$$
Q_{M}=0,25 Q_{I}
$$

Todas as dimensões do modelo físico obedecem a escala de 1:5 em relação ao desgaseificador industrial, exceto o diâmetro dos bicos injetores que foi escolhido de forma a assegurar a igualdade do número de Froude modificado entre o modelo físico e o equipamento industrial. O diâmetro dos bicos injetores do modelo físico $\phi_{M}(\mathrm{~m})$ se relaciona com o diâmetro dos bicos no $\mathrm{RH}$ industrial $\phi_{I}(\mathrm{~m})$ por:

$$
\phi_{M}=0,45 \phi_{I}
$$




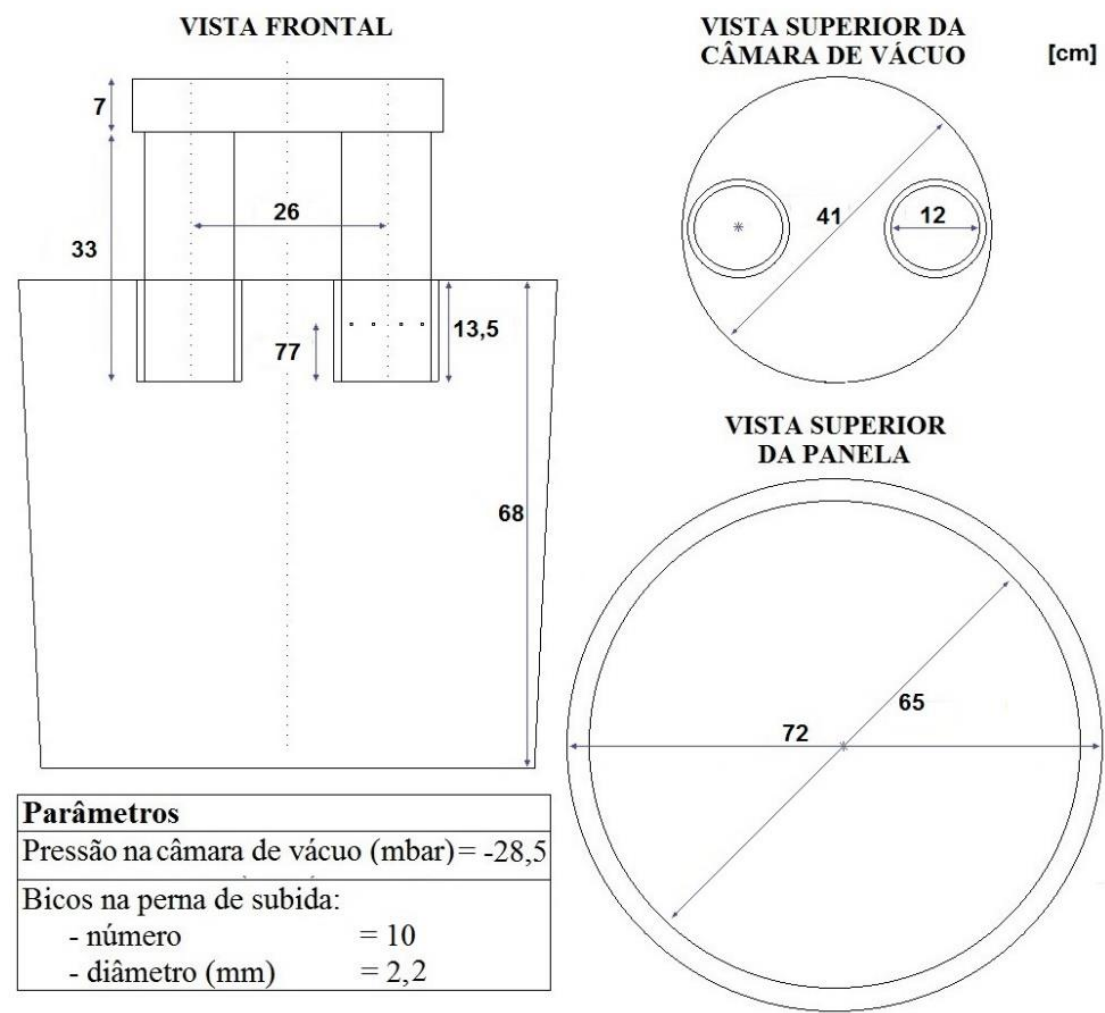

Figura 1. Dimensões de região ocupada inicialmente pela água no modelo físico de desgaseificador $\mathrm{RH}$ estudado.

Embora o modelo físico possua dez bicos injetores de ar, o uso simultâneo de todos os injetores não permite a visualização adequada da penetração do gás na perna subida. Assim, nos experimentos apresentados aqui, foram utilizados apenas um (condição B1) ou dois bicos injetores diametralmente opostos (condição B2). A penetração do jato foi registrada por câmara de vídeo comum. A Figura 2 apresenta um esquema da montagem usada nesse estudo.

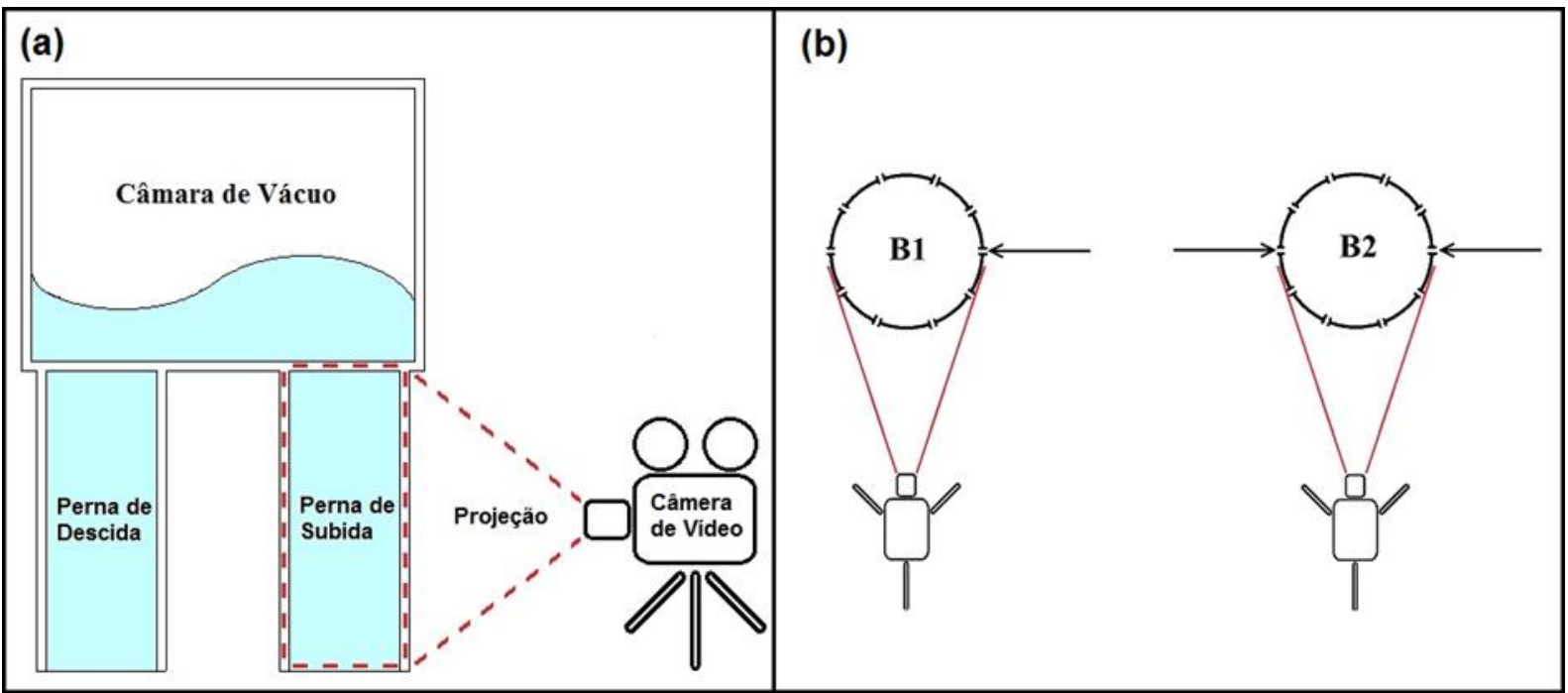

Figura 2. Esquema da montagem usada nos experimentos físicos: (a) região filmada por câmera de vídeo comum; (b) posição dos bicos injetores usados nos dois casos estudados. Condição B1 usa apenas um bico injetor; condição B2 utiliza dois bicos injetores diametralmente opostos. 
Nos experimentos, foram utilizadas vazões de ar de $5 \mathrm{l} / \mathrm{min}, 10 \mathrm{l} / \mathrm{min}, 20 \mathrm{l} / \mathrm{min}, 30 \mathrm{l} / \mathrm{min}$, $40 \mathrm{l} / \mathrm{min}$ e $50 \mathrm{l} / \mathrm{min}$ por bico injetor.

\subsection{Simulações Matemáticas}

A penetração de ar na perna de subida do modelo físico descrito na seção anterior foi predita por um modelo matemático. O modelo matemático utilizado adota a abordagem multifásica euleriana-euleriana, na qual são resolvidas as seguintes equações de conservação [10]:

- Equação de conservação de massa de cada fase, água e ar;

- Equações de conservação de quantidade de movimento de cada fase, água e ar, nas três direções cartesianas ( $x, y$ e $z$ );

- Equação de conservação de volume (soma das frações volumétricas de ar e água igual a 1).

A turbulência de cada fase foi modelada separadamente. Para a fase contínua (água), foi utilizado o modelo k- $\varepsilon$ padrão [11]. Para a fase discreta (ar), foi usado um modelo de turbulência algébrico simples que assume que a fase discreta possui a mesma viscosidade cinemática turbulenta da fase contínua. A transferência de turbulência entre as fases foi desprezada.

A única força de interação entre fases considerada foi a força de arraste. O coeficiente de arraste foi calculado por meio do modelo de Grace com coeficiente de correção de fração volumétrica igual a 2 [4]. O modelo de Grace requer o valor da tensão interfacial água-ar que foi especificada como $0,072 \mathrm{~N} / \mathrm{m}$ [12]. Foi arbitrado diâmetro fixo de bolhas de ar de $3 \mathrm{~mm}$ [4].

$\mathrm{O}$ ar foi tratado como gás ideal. O sistema foi considerado isotérmico a $25^{\circ} \mathrm{C}$ e a pressão ambiente assumida igual a 1atm. O software ANSYS CFX foi usado nos cálculos. As propriedades físicas da agua e ar adotadas nas simulações foram os padrões do software para esses materiais: densidade da água igual a $997 \mathrm{~kg} / \mathrm{m}^{3}$, viscosidade da água igual a $8,899 \times 10^{-4} \mathrm{~Pa} \cdot \mathrm{s}$, massa molar do ar igual a $28,96 \mathrm{~g} / \mathrm{mol}$ e viscosidade do ar igual a $1,831 \times 10^{-5} \mathrm{~Pa} \cdot \mathrm{s}$.

O modelo utilizado é matematicamente similar ao adotado por outros autores [4-6]. Seus principais diferenciais são numéricos.

Foi utilizado esquema de advecção de $2^{2}$ ordem (High Resolution no ANSYS CFX) para todas as equações de conservação, com exceção das equações da modelo turbulência, no qual foi usado esquema de advceção de $1^{\underline{a}}$ ordem. Por ser mais instável que o esquema de $1^{\underline{a}}$ ordem (Upwind no ANSYS CFX), o uso do esquema de $2^{\mathrm{a}}$ ordem exigiu que a simulação fosse executada de maneira transiente. Os resultados apresentados nesse trabalho foram obtidos após 30s de simulação com passo de tempo de 0,01 s utilizando esquema de $2^{\underline{a}}$ ordem para discretização do tempo (Second Order Backward Euler no ANSYS CFX).

Por sua vez, a simulação transiente exige que a superfície livre água-ar existente na câmara de vácuo seja predita pelo método numérico de modo a garantir a conservação da massa inicial de água. Para esse fim, não foi usado nenhum esquema de reconstrução [13] ou compressão de interface [14]. A interface foi capturada e mantida integra apenas pela adoção de uma malha suficientemente refinada (Figura 3 ) e pelo uso do esquema de advecção de $2^{\circ}$ ordem [15]. Devido à simetria do sistema, apenas metade da geometria $\mathrm{RH}$ foi simulada. A malha utilizada possui 724998 nós e 803516 elementos. 


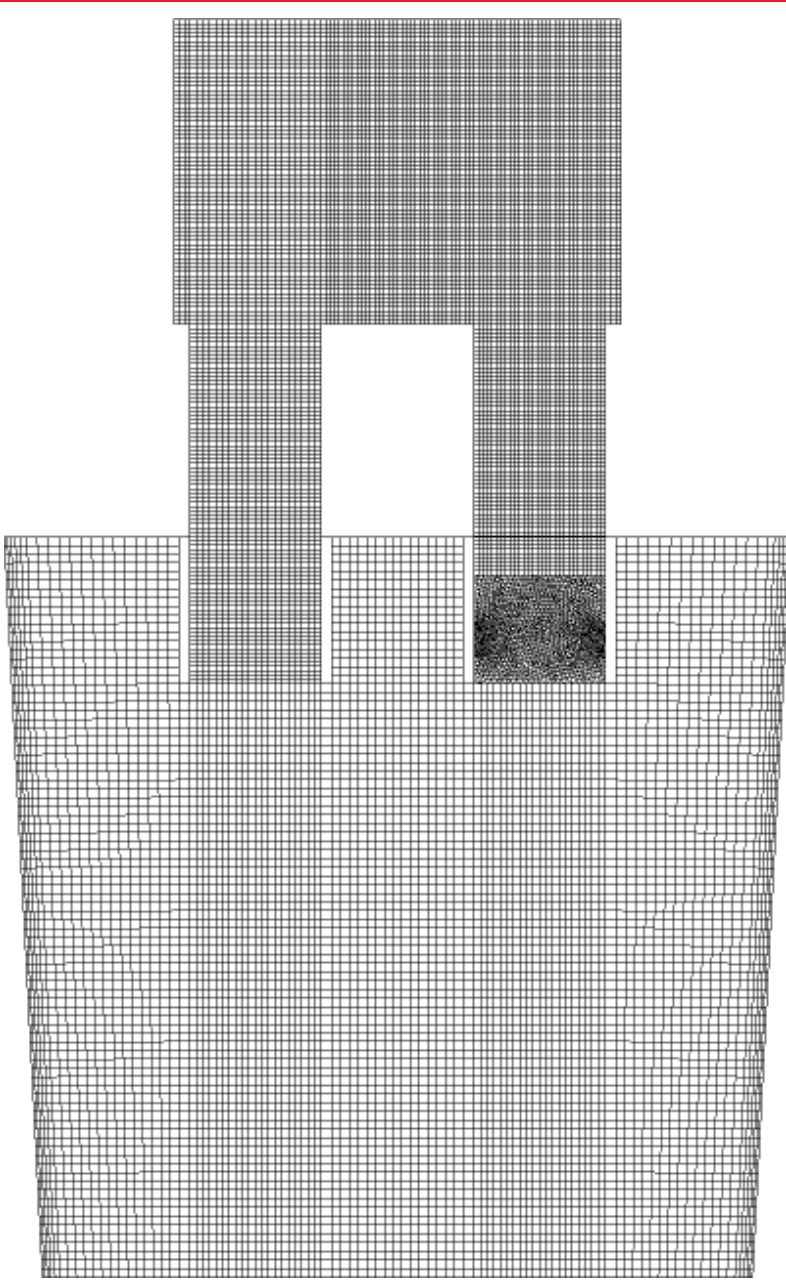

Figura 3. Malha computacional usada nas simulações de penetração de gás no modelo físico de um desgaseificador $\mathrm{RH}$ industrial.

As condições de contorno adotadas no modelo matemático foram:

- Bicos injetores (um ou dois): especificação da vazão de entrada de gás correspondente a cada caso estudado no modelo físico (5l/min, 10l/min, 201/min, 301/min, 401/min e 501/min por bico injetor);

- Paredes da panela, pernas do RH e câmara de vácuo: condição de não escorregamento para a água (uso de função de parede "escalável") e condição de escorregamento livre para o ar;

- Plano de simetria: fluxo nulo de massa, quantidade de movimento e variáveis do modelo de turbulência;

- Superfície livre da panela: condição de escorregamento livre;

- Topo da câmara de vácuo $(280 \mathrm{~mm}$ da altura da câmara de vácuo foram modelados): condição de abertura, que permite saída de ambos os fluidos e entrada apenas de ar, com pressão relativa de - 2591Pa (dada pela coluna de água de $0,265 \mathrm{~m}$ sobre o nível de água da panela) e demais configurações padrões do ANSYS CFX.

Como condição inicial para o caso B1 com vazão de $5 \mathrm{l} / \mathrm{min}$, foi considerada velocidade nula dos fluidos, pressão estática e fração volumétrica de fases corresponde à condição posterior à aplicação do vácuo e anterior ao começo da injeção de gás. As condições iniciais dos demais casos B1 foram tomados como a solução final da vazão imediatamente inferior. A condição inicial do caso B2 com vazão de $51 / \mathrm{min}$ por bico foi 
escolhida como a solução final do caso B1 para a mesma vazão. A condição inicial das demais vazões do caso B2 seguiram regra análoga a do caso B1.

Em todas as simulações, foi estipulado como critério de convergência valor RMS dos resíduos (adimensionalizados) das equações de conservação menor que $10^{-5}$. O valor RMS (Root Mean Square) dos resíduos corresponde à raiz quadrada da soma dos resíduos de cada volume de controle do domínio elevado ao controle. Esse critério foi buscado por até 10 iterações (Coefficient Loops no ANSYS CFX) a cada passo de tempo.

\section{RESULTADOS E DISCUSSÃO}

A Figura 4 apresenta os resultados obtidos experimentalmente, no modelo físico, e as previsões correspondentes fornecidas pelo modelo matemático.

Para os casos B1, com apenas um bico injetor, os resultados obtidos pelas duas abordagens concordam qualitativamente tanto em relação à penetração do gás quanto à sua dispersão. Contudo a dispersão do gás é subestimada para a vazão de gás de $5 \mathrm{l} / \mathrm{min}$.

Já as previsões para os casos B2, com dois bicos injetores, concordam qualitativamente apenas para vazões de até $30 \mathrm{l} / \mathrm{min}$. Para vazões maiores, os resultados experimentais exibem grande dispersão de bolhas de gás enquanto os resultados matemáticos predizem a concentração progressiva de gás na região central da perna de subida à media que vazão de gás é aumentada. A penetração de gás é também subestimada pelo modelo matemático. Adicionalmente, não é claro se as oscilações exibidas pelo modelo matemático para a vazão de 50l/min apresentam qualquer significado físico.

Possivelmente, as diferenças observadas se devem aos modelos simples de turbulência e de interação de fases adotados. Outra fonte de incerteza são desvios das dimensões reais do modelo físico em relação às suas dimensões nominais, adotadas pelo matemático. É sugerido o desenvolvimento de trabalhos futuros para o aprimoramento do modelo matemático.

Apesar das falhas do modelo matemático discutidas acima, é aparente que o problema de penetração de ar encontrado na literatura não está relacionado com a atuação da força de massa virtual, como sugerido por Geng et al. [8], ou outros mecanismos físicos. Neves [4] também não identificou melhora significativa da penetração de ar com introdução das forças sustentação, dispersão turbulenta e lubrificação da parede.

A fim de elucidar a fonte do problema de penetração de ar, um modelo matemático simplificado foi utilizado. Esse considera regime estacionário e interface água-ar presente na câmara de vácuo como uma condição de contorno (escorregamento livre para água e saída para o ar). Esse modelo é numericamente estável para todos as vazões estudadas da condição B1 e para vazões de até $10 \mathrm{l} / \mathrm{min}$ por bico da condição B2. Por causa disso, foi adotada a simulação transiente no restante do presente trabalho.

A Figura 5 compara resultados dos dois tipos de abordagens para o caso B1 com vazão de $50 \mathrm{l} / \mathrm{min}$. A previsão da previsão da taxa de circulação pelos modelos matemáticos de $\mathrm{RH}$ foi afetada pela forma de modelagem da interface água-ar na câmara de vácuo, em conformidade com trabalho recente de Costa e Tavares [5].

A Figura 6 apresenta previsões do modelo matemático estacionário para a condição B1 com vazão de gás de 50l/min. A primeira simulação utiliza esquema de advecção de $1^{\text {a }}$ ordem (upwind). A segunda simulação também usa esquema de advecção de 
$1^{\text {a }}$ ordem (upwind), mas utiliza uma malha substancialmente mais refinada (462004 nós contra 77297 nós). A terceira simulação utiliza a mesma malha refinada da segunda simulação, mas usa esquema de advecção de $2^{\underline{a}}$ ordem (High Resolution).

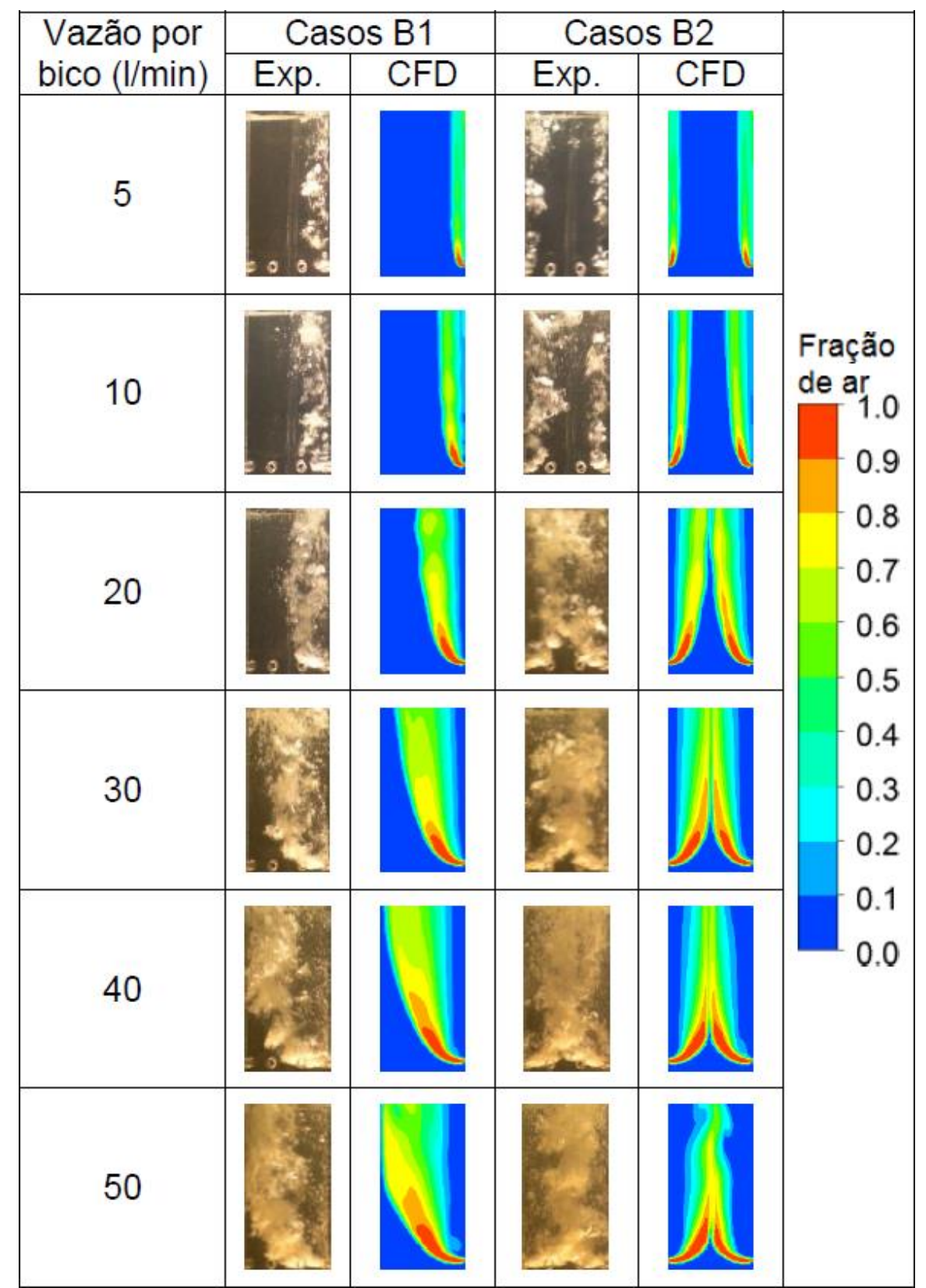

Figura 4. Comparação entre resultados experimentais (Exp.) e as previsões do modelo matemático (CFD) após 30s de simulação. Nos casos B1, apenas um bico injetor é usado. Nos casos B2, dois bicos injetores são utilizados. Os resultados físicos correspondem a quadros da filmagem da perna de subida do modelo físico feita em acrílico. Os resultados matemáticos são mapas de contorno plotados sobre o plano de simetria da perna de subida.

A previsão obtida na simulação que utiliza esquema de advecção de $1^{\text {a }}$ ordem e malha grosseira é similar ao apresentado na literatura exibindo ar apenas próximo à parede em que é feita a injeção de gás. Mantendo o uso do esquema de advecção de $1^{a}$ ordem, o refino substancial de malha é capaz de melhorar um pouco a penetração do gás. Já a adoção do esquema de advecção de $2^{\mathfrak{a}}$ ordem em conjunto com a malha refinada possibilita a previsão de penetração de ar qualitativamente similar àquela observada experimentalmente (Figura 5). Fica claro que o problema de penetração de 
ar se deve a erros numéricos relacionados à discretização insatisfatórias das equações que governam o escoamento no desgaseificador $\mathrm{RH}$.

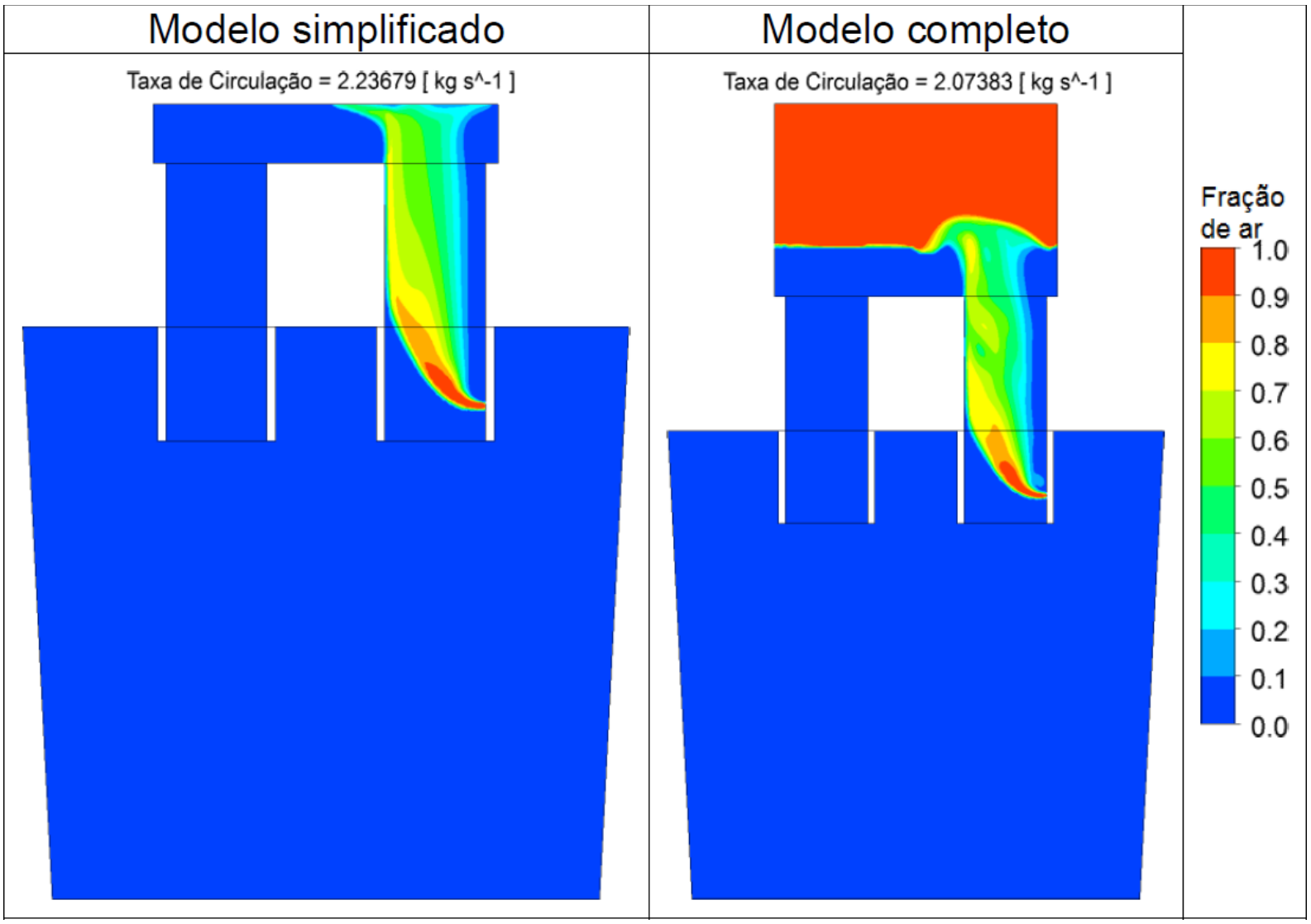

Figura 5. Comparação entre previsões do modelo simplificado (estacionário) e do modelo completo (transiente). Os resultados matemáticos são mapas de contorno plotados sobre o plano de simetria do desgaseificador $\mathrm{RH}$.

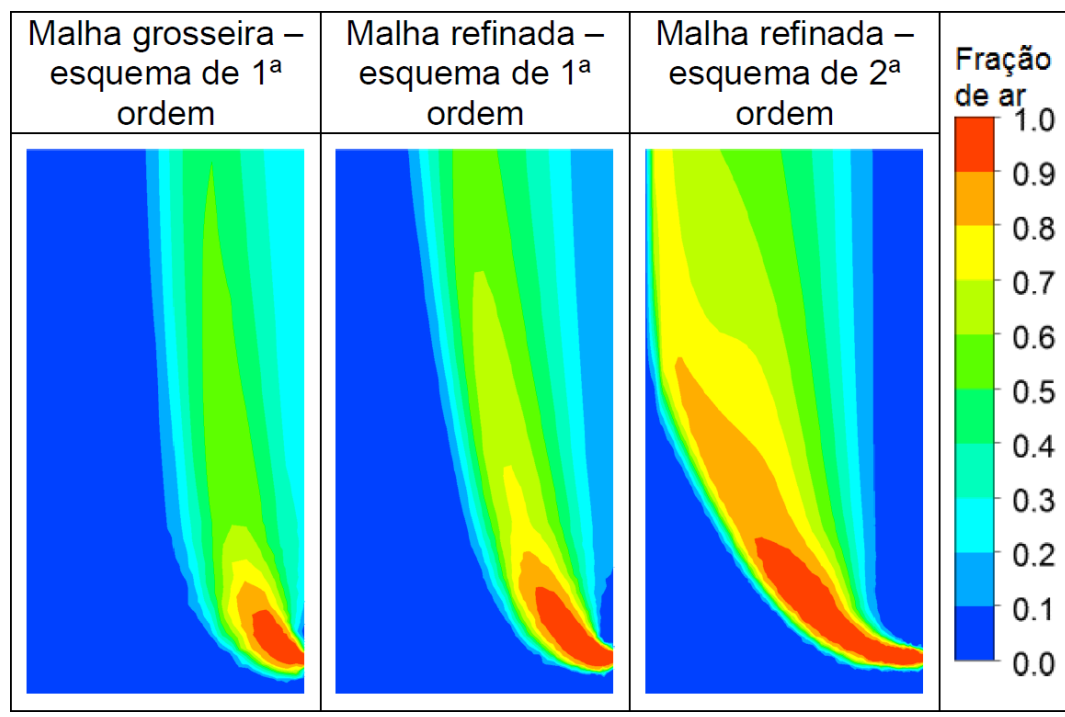

Figura 6. Previsões do modelo matemático estacionários para o caso B1 com vazão de $50 \mathrm{l} / \mathrm{min}$. Os resultados matemáticos são mapas de contorno plotados sobre o plano de simetria da perna de subida.

As taxas de circulação preditas para os modelos da Figura 5 são 2,44kg/s (malha grosseira e esquema de $1^{\text {a }}$ ordem), $2,58 \mathrm{~kg} / \mathrm{s}$ (malha refinada e esquema de $1^{\text {a }}$ ordem) e $2,24 \mathrm{~kg} / \mathrm{s}$ (malha refinada e esquema de $2^{\mathrm{a}}$ ordem). Esses resultados diferem 
significativamente da taxa de circulação predita pelo modelo completo (Figura 4), $2,07 \mathrm{~kg} / \mathrm{s}$.

A abordagem numérica proposta é computacionalmente custosa e seu emprego pode ser proibitivo devido a restrições de tempo e custo. Entretanto, ela é chave para se obter resultados mais acurados. De acordo com as recomendações da AIAA [16], todas as simulações CFD devem ser adotar esquemas numéricos de, pelo menos, $2^{a}$ ordem.

\section{CONCLUSÃO}

A penetração de gás na perna de subida de desgaseificador $\mathrm{RH}$ foi investigada por meio de modelos físico e matemático. Foram estudadas casos com um ou dois bicos injetores e vazões de gás por bico de $5 \mathrm{l} / \mathrm{min}, 10 \mathrm{l} / \mathrm{min}, 20 \mathrm{l} / \mathrm{min}, 30 \mathrm{l} / \mathrm{min}, 40 \mathrm{l} / \mathrm{min}$ e $501 / \mathrm{min}$.

Para as condições com um bico injetor, os resultados obtidos pelas duas abordagens apresentaram boa concordância qualitativa, embora a dispersão de gás foi subestimada para a vazão de $5 \mathrm{l} / \mathrm{min}$. Já para as condições com dois bicos injetores, os resultados do modelo matemático só concordaram qualitativamente com os experimentos físicos para vazões de até 30l/min. Para vazões maiores, o gás tendeu a se concentrar no centro da perna no modelo matemático enquanto que 0 experimento físico indica a dispersão do gás por toda a perna.

Adicionalmente, os resultados indicam que o problema de penetração de gás identificado na literatura está relacionado com erros numéricas e não com falhas na modelagem da física do problema.

\section{Agradecimentos}

Os autores agradecem o apoio financeiro da FAPEMIG - Fundação de Amparo à Pesquisa do Estado de Minas Gerais, Brasil - na forma da bolsa de pesquisa para R. Tavares, Processo № PPM-00118-13. Os autores também agradecem o apoio da CAPES/PROEX ao programa de pós-graduação PPGEM/UFMG e a bolsa de doutorado, no 1487157, concedida pela CAPES a B. Braga.

\section{REFERÊNCIAS}

1 Ghosh A. Secondary steelmaking: principles and applications. Boca Raton: CRC Press; 2000.

2 Kor GJW, Glaws PC. Ladle refining and vacuum degassing. In: Fruehan RJ. The making, shaping and treating of steel, vol. 2: steelmaking and refining volume. $11^{\mathrm{a}}$ edition. Pittsburgh: The AISE Steel Foundation; 1998.

3 Silveira WJ. Modelamento matemático do escoamento de fluidos no processo $\mathrm{RH}$ para previsão da taxa de circulação do aço [dissertação de mestrado]. Belo Horizonte: UFMG; 2011.

4 Neves L. Modelagem do escoamento multifásico no desgaseificador $\mathrm{RH}$ e no distribuidor de lingotamento contínuo [tese de doutorado]. Belo Horizonte: UFMG; 2012.

5 Costa LT, Tavares RP. Dessulfuração do aço em desgaseificador RH por meio de modelo matemático. In: Associação Brasileira de Metalurgia, Materiais e Mineração. Anais do 46은 Seminário de Aciaria - Internacional; 2015; Rio de Janeiro, Brasil. São Paulo: ABM; 2015.

6 Silva TC, Rodrigues EF, Soares C, Da Silva CA, Da Silva IA. Simulação numérica do fluxo recirculatório em um reator $\mathrm{RH}$ : aplicação à dessulfuração. In: Associação 
Brasileira de Metalurgia, Materiais e Mineração. Anais do 46은 Seminário de Aciaria Internacional; 2015; Rio de Janeiro, Brasil. São Paulo: ABM; 2015.

7 Geng D-Q, Zheng J-X, Wang K, Wang P, Liang R-Q, Liu H-T, et al. Simulation on decarburization and inclusion removal process in the Ruhrstahl-Heraeus $(\mathrm{RH})$ process with ladle bottom blowing. Metallurgical and Materials Transactions B. 2015;46(3):14841493.

8 Geng D-Q, Lei H, He J-C. Numerical simulation of the multiphase flow in the RheinsahlHeraeus $(\mathrm{RH})$ system. Metallurgical and Materials Transactions B. 2010;41(1):234-247.

9 Neves L. Avaliação dos efeitos da injeção de gás na câmara de vácuo do modelo físico de um desgaseificador RH [dissertação de mestrado]. Belo Horizonte: UFMG; 2008.

10 ANSYS CFX-solver theory guide, 14.5. Canonsburg: ANSYS; 2012.

11 Launder BE, Spalding DB. The numerical computation of turbulent flows. Computer Methods in Applied Mechanics and Engineering. 1974;3(2):269-289.

12 Incropera FP, Dewitt DP, Bergman TL, Lavine AS. Fundamentals of Heat and Mass Transfer. 6 ${ }^{\mathrm{a}}$ edition. Hoboken: Willey; 2006.

13 Glitz KLZ. Modelagem numérica de parâmetros da interface e sua aplicação na simulação do efeito Jamin [tese de doutorado]. Florianópolis: UFSC; 2012.

14 Hänsch SH, Lucas D, Höhne T, Krepper E, Montoya G. Comparative simulations of free surface flows using VOF-methods and a new approach for multi-scale interfacial structures. In: American Society of Mechanical Engineers. Proceedings of the ASME 2013 Fluids Engineering Division Summer Meeting; 2013; Incline Village, EUA. Nova lorque: ASME; 2013.

15 Rezende RVP. Modelagem matemática e desenvolvimento de metodologia computacional para a simulação numérica do escoamento bifásico de ar e ferro-gusa em canal de corrida de alto-forno [dissertação de mestrado]. Florianópolis: UFSC; 2008.

16 Guide for the verification and validation of computational fluid dynamics simulations. Reston: AIAA; 1998. 